\title{
A coarse-grain model of growth and cell cycle in Saccharomyces cerevisiae: a mathematical analysis
}

\author{
Pasquale Palumbo ${ }^{1,3 *}$, Federico Papa ${ }^{1,2 *}$, Marco Vanoni ${ }^{1,3}$, \\ Lilia Alberghina ${ }^{1,3}$
}

${ }^{1}$ SYSBIO/ISBE.IT, Centre of Systems Biology

2 CNR-IASI via dei Taurini 19, Italian National Research Council (CNR), 00168 Rome, Italy

${ }^{3}$ Department of Biotechnology and Biosciences, University of Milano-Bicocca, Piazza della Scienza, Milan, Italy

Corresponding author: pasquale.palumbo@unimib.it;

federico.papa@iasi.cnr.it, \{marco.vanoni, lilia.alberghina\}@unimib.it

*These authors contributed equally to this work.

\begin{abstract}
In this paper a coarse-grain model is presented that describes the major features of cell growth and cell cycle in Saccharomyces cerevisiae. Central for the construction of the growth and cell cycle model has been the large amount of scientific papers covering the description of cellular growth in steady-state and perturbed growth conditions, describing ribosome and protein contents, and the description of cell cycle progression as percentage of budded cells (cells that have entered S-phase). The coarse model is composed by i) a growth module, i.e. a set of ODEs representing the dynamics of synthesis/degradation of ribosomes and proteins, and ii) a cell cycle module, i.e. a set of three consecutive timers $\left(T_{1}, T_{2}\right.$ and $\left.T_{B}\right)$ that temporally accounts for the yeast cell cycle, underlying the length of the G1 phase (timer $T_{1}$ plus $T_{2}$ ) and of the budded phase (timer $T_{B}$ ) entailing $S, G 2$ and $M$ phases. The growth module acts as a master, setting the length of the first of the three sequential timers. Main results coming from the mathematical analysis involve the qualitative behavior of the system, constraining ribosome synthesis and growth to the set of model parameters. Further results involve the generalization of a known constraint that involves the lengths of the cycles of parent and daughter cells, and accounts for the genealogical age heterogeneity, typical of budding yeast Saccharomyces cerevisiae.
\end{abstract}

Keywords: Linear ODE models, Systems Biology.

\section{Introduction}

This work investigates the qualitative behavior of a coarse-grain mathematical model of the budding yeast Saccharomyces cerevisiae, a micro-organism known to be exploited as a model for eukaryotic cells. The model constitutes of two modules. The 
former describes the cell growth by means of a pair of Ordinary Differential Equations (ODEs) dealing with ribosomes and protein content. The latter introduces a set of three timers that cover the whole cell cycle, namely $T_{1}, T_{2}$ and $T_{B}$. Timer $T_{1}$ starts with the newborn cell and is formally over when the G1/S regulon is activated; then $T_{2}$ starts and is over with the end of the G1 phase. The notation used for the first two timers $T_{1}$ and $T_{2}$ is the same one that has been introduced in [1]. According to the mentioned paper, $T_{1}$ refers to the period a newborn cell takes to activate the G1/S regulon and is formally measured by the time the regulon inhibitor Whi5 takes to exit the nucleus; on the other hand, $T_{2}$ refers to the time cyclins Clb5/6 (responsible for the onset of the $\mathrm{S}$ phase) take to get rid of their inhibitor Sic1. The sum of $T_{1}$ and $T_{2}$ provides the length of the G1 phase.

At the end of the G1 phase, contemporary to the onset of the $\mathrm{S}$ phase, $T_{B}$ starts and covers the rest of the cycle, i.e. $\mathrm{S}+\mathrm{G} 2+\mathrm{M}$ phases. Such a period is called budded phase because the cell is characterized by a bud, and all protein and ribosome produced in $T_{B}$ go straightforwardly to the bud (that will become the newborn cell at the end of the cycle).

Growth and cell cycle are linked together by timer $T_{1}$, since its length depends on the cell size. More in details, the link is rendered by the fact that $T_{1}$ depends on the initial cell size, i.e. its length has an inverse dependence on the protein content at cell birth. This is a simplifying hypothesis with respect to the more accurate model developed in [2], where the link between growth and cell cycle is driven by a molecular sizer, that is able to account also for extra-small cell.

Figure 1 provides a graphical representation of the coarse-grain model, showing the details of each module and the connection point between them.

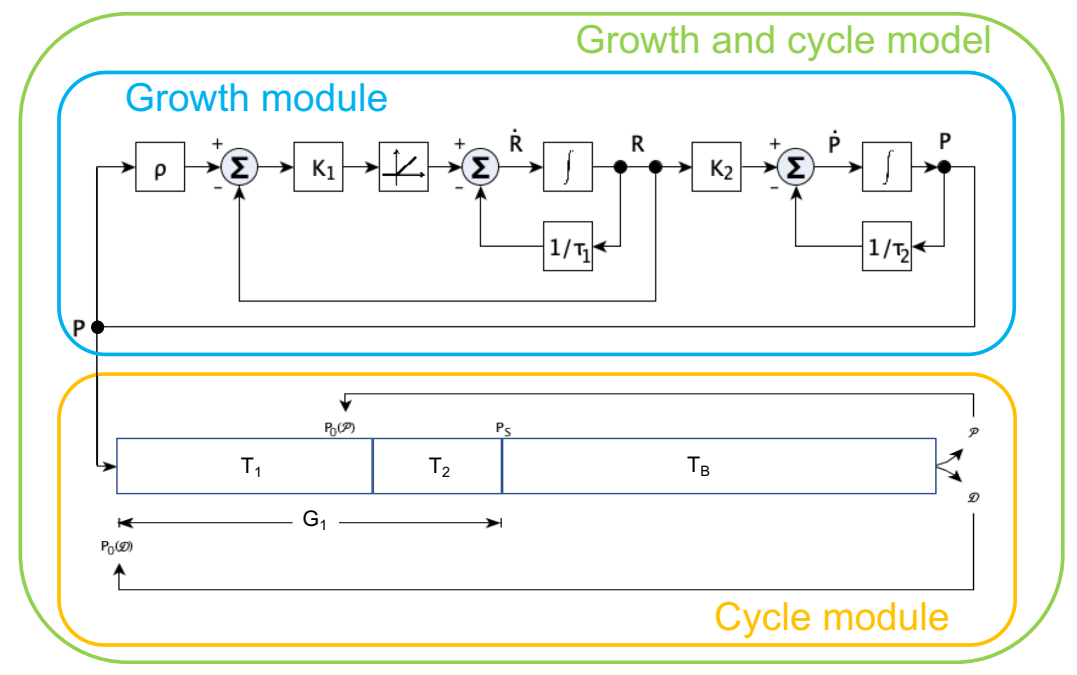

Figure 1

Block diagram of the growth and cell cycle model. 
Part of the mathematical analysis dealing with the growth module has been developed in [3]. Here we extend those results and introduce a set of constraints straightforwardly related to the length of parent and daughter cycles.

\section{The growth and cell cycle model}

The growth and cell cycle model is composed of two modules (see Figure 1):

- the growth module based on a set of ordinary differential equations that describes the dynamics of synthesis and degradation of ribosomes and proteins;

- the cell cycle module consisting of a sequence of the three timers $T_{1}, T_{2}$ and $T_{B}$ that describes the cell cycle progression after cell birth.

\subsection{The growth module}

Cell proliferation is sustained by the increase of cell components. A large part of energy and building blocks utilized in cellular processes is exploited for the biosynthesis of ribosomes and proteins, whose increase results from the balance between the rate of protein/ribosome synthesis and degradation.

The growth module is taken from [4] and deals with the ribosome content $R$, expressed as number of ribosomes per cell, and the protein content $P$, expressed as number of polymerized amino acids per cell. The working hypothesis is that ribosome and protein contents are allowed to vary continuously, so that the dynamics of the two state variables is described by the following ODEs:

$$
\begin{cases}\dot{R}(t)=K_{1}[\rho P(t)-R(t)]^{+}-\frac{R(t)}{\tau_{1}}, & R(0)=R_{0} \geq 0, \\ \dot{P}(t)=K_{2} R(t)-\frac{P(t)}{\tau_{2}}, & P(0)=P_{0} \geq 0,\end{cases}
$$

where

$[z]^{+}= \begin{cases}z, & \text { for } z>0 \\ 0, & \text { otherwise }\end{cases}$

Both ribosome and protein dynamics are described by the balance between production and degradation rates. A special role is played by the parameter $\rho$, that represents the ideal "ribosomes-over-proteins" ratio, for each steady-state growth condition. When the ratio $R(t) / P(t)$ is higher than $\rho$, then there is no ribosome production, so that the dynamics of $R$ and $P$ is described by the following linear working mode,

$\mu_{1}: \quad\left\{\begin{array}{l}\dot{R}(t)=-\frac{R(t)}{\tau_{1}}, \\ \dot{P}(t)=K_{2} R(t)-\frac{P(t)}{\tau_{2}} ;\end{array}\right.$

otherwise, the ribosome production rate is proportional to the (positive) difference $\rho P(t)-R(t)$ by means of parameter $K_{1}$ and the $R-P$ dynamics is described by an- 
other linear working mode as follows

$\mu_{2}: \quad\left\{\begin{array}{l}\dot{R}(t)=-\left(K_{1}+\frac{1}{\tau_{1}}\right) R(t)+K_{1} \rho P(t), \\ \dot{P}(t)=K_{2} R(t)-\frac{P(t)}{\tau_{2}} .\end{array}\right.$

The switches between $\mu_{1}$ and $\mu_{2}$ depend on the position of the state variables of the system in the phase plane and, in particular, on the value of their ratio $R(t) / P(t)$ compared to the threshold $\rho$ (see Figure 2 where an example of trajectory in the phase plane is reported).

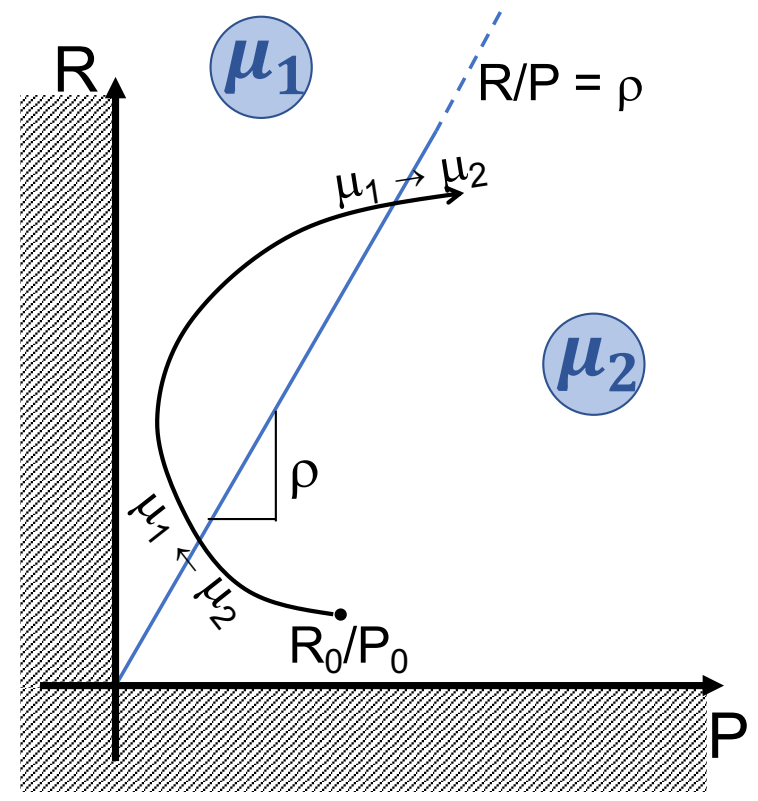

Figure 2

An explanatory trajectory in the phase-plane for a cell that is born according to ribosome synthesis conditions, then it first switches to absence of ribosome synthesis (from $\mu_{2}$ to $\mu_{1}$ ) and then it switches back to $\mu_{2}$ again.

The properties of each working mode and the switching conditions from one mode to the other one are given in Section 3 and are partially demonstrated in [3]. Such properties are necessary to characterize the qualitative behaviour of $R(t)$ and $P(t)$, as well as to establish if the yeast cell is actually growing (i.e. if the linear model has at least one positive eigenvalue) or not.

Experimental evidences related to yeast populations in exponential growth show that each growth condition (i.e. each growth rate) is characterized by a specific ratio of "ribosomes-over-proteins", although the molecular mechanism of the association is still unclear. The mechanism may involve TOR-dependent phosphorylation of Sfp1, a positive regulator of transcription of genes encoding ribosomal proteins [5]. 
Experimental evidences also support the choice of the non-linear ribosome production rate of model (1) as they show the existence of a negative feedback reducing the ribosome biosynthesis in presence of ribosomes not engaged in protein biosynthesis [6,7]. In Escherichia coli, this mechanism runs via ppGpp and is relatively well understood; it appears to provide a robust and optimal partitioning of cellular resources over ribosomes and other proteins [8]. In eukaryotes it involves the TOR pathway $[5,9,10]$. Experimental values for the "ribosomes-over-proteins" $\rho$ can be found in [11-14].

\subsection{The cell cycle module}

Saccharomyces cerevisiae cells divide asymmetrically [15], cell mass at division is unequally partitioned between a larger, old parent cell $(\mathscr{P})$ and a smaller, newly synthesized daughter cell ( $\mathscr{D})$. The degree of asymmetry of cell division in Saccharomyces cerevisiae is modulated by nutrients: poor media - such as ethanol - yield a high level of asymmetry with large parent cells and very small daughter cells, whereas in rich media - such as glucose - parents and daughters at division are very close in size (reviewed in [16]). Since cells have to grow to a critical cell size before entering $\mathrm{S}$ phase and budding, small cells have longer cycle time than larger cells, most notably in poor media. As a matter of fact, this difference in cycle time is due to differences in the G1 phase, having the budded period $T_{B}$ essentially the same length whatever the size of the cells [1].

The first timer $T_{1}$ starts with the birth of the new cell and is over when nuclear Whi5 exits the nucleus. Aiming not to detail the whole molecular machinery, Whi5 will not be explicitly involved in the model. The length of $T_{1}$ is strongly related to the initial size of the cell, according to a constraint that makes smaller the length of $T_{1}$ for larger cells and vice versa. So, the initial size of the cell plays a crucial role to assess the value of $T_{1}$. More in details, $T_{1}$ is set according to the following equation

$T_{1}=\max \left\{T_{1 P}, W_{0}-W_{1} \ln (P(0))\right\}$,

with $P(0)$ denoting the size of the cell at birth. Notice that $P(0)$ plays an active role in the setting of $T_{1}$ only for cells small enough to ensure

$W_{0}-W_{1} \ln (P(0))>T_{1 P} \quad \Longrightarrow \quad P(0)<e^{\frac{W_{0}-T_{1 P}}{W_{1}}}$.

Length of timer $T_{2}$ does not depend on protein content, and it is set to the same value for small and large cells. At the end of timer $T_{2}$, the critical size expressed both as ribosome content and as protein content, $R_{S}$ and $P_{S}$ respectively, is estimated. Timer $T_{2}$ is related to the period inhibitor Sic1 takes to get out of the nucleus thus activating cyclins Clb5,6, responsible for the onset of the budded phase [1].

The third timer $T_{B}$ refers to the budded period, which eventually leads to cellular division. Like timer $T_{2}, T_{B}$ length does not depend on the protein content, and it is set to the same value for small and large cells.

Notice that the setting of timer $T_{1}$ may allow small cells (i.e. the ones that comply with (5)) to reduce their critical cell size variability w.r.t. the initial variability. 
Indeed, denote with $\bar{P}_{0}$ and $\Delta_{0}$ the average value of the initial size and its corresponding variability in a population of cells in balanced exponential growth (growth rate $\lambda$ ), with the initial size complying with inequality (5). Define $\bar{P}_{s}$ and $\Delta_{s}$ the average value of the critical size and its corresponding variability. Then, because of (5), we have:

$P_{S}=P(0) e^{\lambda\left(T_{1}+T_{2}\right)}=P(0) e^{\lambda\left(W_{0}-W_{1} \ln P(0)+T_{2}\right)}$,

By accounting for size fluctuations, we obtain after simple computations:

$P_{S}=\bar{P}_{s}+\Delta_{s}=\left(\bar{P}_{0}+\Delta_{0}\right)^{1-\lambda W_{1}} e^{\lambda\left(W_{0}+T_{2}\right)}$.

Applying first-order approximation, we finally have

$\Delta_{s} \simeq\left(1-\lambda W_{1}\right) \bar{P}_{0}^{-\lambda W_{1}} e^{\lambda\left(W_{0}+T_{2}\right)} \Delta_{0}$.

A biologically meaningful parameter setting provides $\Delta_{s} \leq \Delta_{0}$ (see for instance [2]), which is coherent with the idea that the G1/S transition is able to reduce size variability.

\subsection{Genealogical age heterogeneity}

When a yeast cell buds, a chitin ring, called bud scar, builds up at the bud isthmus and remains on the parent cell after the bud has separated (reviewed in [16]). Since each new bud starts at a new site, it is possible to determine the number of bud scars $s$ present on the surface of a parent cell and consequently to establish the genealogical age $k$ of the parent cell, meaning the age of the parent cell equal to the number of daughters it has generated (i.e., $k=s$ ). So, denoting by $\mathscr{P}_{k}$ a parent cell of age $k$, a cell $\mathscr{P}_{1}$ has one bud scar since it has completed a cycle, a cell $\mathscr{P}_{2}$ has two bud scars since it has completed two cycles, and so on. On the other hand, a cell without bud scars $(s=0)$ is a daughter cell and it has not yet completed a cycle. The model of growth and cell cycle, however, distinguishes the genealogical age of the daughter cells: it can be 1 if the daughter is born from another daughter, while it is $k>1$ if the daughter is born from a parent $\mathscr{P}_{k-1}$. We denote by $\mathscr{D}_{k}$ a daughter of genealogical age $k$. It has to be stressed that such a notation refers to a cell in a specific cycle of its life. In other words, the same cell is labeled by a different name each time a new cycle starts. For instance, a newborn cell coming from any daughter cell is called $\mathscr{D}_{1}$ in its first cycle, will be called $\mathscr{P}_{1}$ in its second cycle, $\mathscr{P}_{2}$ in its third cycle, and so on.

Because at every generation each parent increases in size before starting to bud [17-19] and at division it receives the mass it had at budding (the mass synthesized during the budded phase going to the newborn daughter), it follows that in parents, the critical size increases with genealogical age. Experimental evidence shows that the higher is the genealogical age (i.e. the number of bud scars), the smaller is the increase in size at budding from one generation to the other $[16,20]$. The reduction in cell size increase with genealogical age has been explained by the mechanical stress of the cell wall, which increases with cell size [21,22]. 
In order to account for the aforementioned behavior, both $K_{2}$ and $\tau_{2}$ in Eqs. (1) (rate of protein synthesis and time constant of protein degradation respectively) are decreased to lower and lower values during the pre-budded period (G1 phase, i.e. $T_{1}+T_{2}$ ), according to the parent genealogical age. We define $K_{2 k}$ and $\tau_{2 k}$ the $K_{2}$ and $\tau_{2}$ parameters for a parent cell with genealogical age $k$. At the end of timer $T_{2}$ - coincident with the end of the G1 phase and with the onset of the budded phase - the values of $K_{2 k}$ and $\tau_{2 k}$ return to the nominal values of $K_{2}$ and $\tau_{2}$, so that the parent cell $\mathscr{P}_{k}$ grows again with the steady-state exponential rate (given by the positive eigenvalue, see next section). Daughter cells (of any genealogical age) are not affected by such a mechanical stress.

Table 1 collects all the model parameters introduced in this section, providing also the corresponding measurement units and definitions.

\begin{tabular}{|c|c|c|}
\hline Parameter & Unit & Definition \\
\hline$\rho$ & rib/aa & Asymptotic ratio of ribosomes over proteins \\
\hline$K_{1}$ & $\min ^{-1}$ & Ribosome production rate \\
\hline$\tau_{1}$ & $\min$ & Ribosome degradation time constant \\
\hline$K_{2}$ & $\mathrm{aa} /(\mathrm{rib} * \min )$ & $\begin{array}{l}\text { Protein production rate } \\
\text { for any } \mathscr{D}_{k} \text { and for } \mathscr{P}_{k} \text { in budded phase }\end{array}$ \\
\hline $\begin{array}{c}K_{2 k} \\
k=1,2, \ldots\end{array}$ & $\mathrm{aa} /\left(\mathrm{rib}^{*} \min \right)$ & $\begin{array}{l}\text { Protein production rate } \\
\text { for } \mathscr{P}_{k} \text { in } \mathrm{G} 1 \text {-phase }\end{array}$ \\
\hline$\tau_{2}$ & $\min$ & $\begin{array}{c}\text { Protein degradation time constant } \\
\text { for any } \mathscr{D}_{k} \text { and for } \mathscr{P}_{k} \text { in budded phase }\end{array}$ \\
\hline $\begin{aligned} & \tau_{2 k} \\
k= & 1,2, \ldots\end{aligned}$ & $\min$ & $\begin{array}{l}\text { Protein degradation time constant } \\
\text { for } \mathscr{P}_{k} \text { in G1-phase }\end{array}$ \\
\hline$T_{1 P}$ & $\min$ & Minimum value for $T_{1}$ \\
\hline$W_{0}$ & $\min$ & $T_{1}$ length for unitary $P(0)$ \\
\hline$W_{1}$ & $\min$ & Size-related coefficient to set $T_{1}$ length \\
\hline$T_{2}$ & $\min$ & Length of $T_{2}$ \\
\hline$T_{B}$ & $\min$ & Length of the budded phase \\
\hline
\end{tabular}

Table 1

Measurement units and definitions of the model parameters.

\section{Properties of the growth module}

It is important to determine which are the conditions on the model parameters of system (1) required for cell growth, under each working mode $\mu_{1}$ or $\mu_{2}$. So, let us introduce the notation $g$ and $\bar{g}$ to denote two opposite growing dynamics of the cell: the state $g$ represents growth, i.e. ribosomes and proteins are actually increasing (after a transient period) because of the existence of a positive eigenvalue in system (1); conversely, the state $\bar{g}$ represents a not growing cell in which ribosomes and proteins are non-increasing.

Let us now give some simple results on the growing dynamics related to system (1). Let us observe first that $\bar{g}$ (no growth) is the only allowed dynamics for system (1) 
when $R(t) / P(t) \geq \rho$, i.e. when the working mode is $\mu_{1}$ (no ribosome synthesis). This trivially comes from the negative sign of the eigenvalues related to the linear system (2) $\left(\lambda_{1}=-1 / \tau_{1}, \lambda_{2}=-1 / \tau_{2}\right)$.

The following theorem shows instead the growing dynamics of system (1) when $R(t) / P(t)<\rho$.

Theorem 1. When the working mode of system (1) is $\mu_{2}$ (presence of ribosome synthesis), both the growing dynamics are allowed. In particular it is:

- $g$ (growth) when $x>0$,

- $\bar{g}$ (no growth) when $-1 \leq x \leq 0$,

where $x$ is the following function of the model parameters

$x=4 \frac{K_{1} K_{2} \rho-\left(K_{1}+\frac{1}{\tau_{1}}\right) \frac{1}{\tau_{2}}}{\left(K_{1}+\frac{1}{\tau_{1}}+\frac{1}{\tau_{2}}\right)^{2}}$.

The condition $x<-1$ cannot occur for any non-negative setting of the model parameters.

Proof. The proof comes from the computation of the eigenvalues of the linear system (3) (working mode of system (1) when $R(t) / P(t)<\rho$ ), that is

$\lambda_{1}=y(-1-\sqrt{1+x}), \quad \lambda_{2}=y(-1+\sqrt{1+x})$,

where $x$ is given by (9) and

$y=\frac{1}{2}\left(K_{1}+\frac{1}{\tau_{1}}+\frac{1}{\tau_{2}}\right)$.

Let us prove first that the condition $x<-1$ cannot be satisfied so that $\lambda_{1}, \lambda_{2}$ are always real (no oscillatory dynamics). In particular, it is shown by the following arguments that $x \geq-1$ for any non-negative setting of the model parameters.

Let us rewrite the quantity $x$ as

$x=t_{1}+t_{2}$,

where

$t_{1}=4 \frac{K_{1} K_{2} \rho}{\left(K_{1}+\frac{1}{\tau_{1}}+\frac{1}{\tau_{2}}\right)^{2}}, \quad t_{2}=-4 \frac{\left(K_{1}+\frac{1}{\tau_{1}}\right) \frac{1}{\tau_{2}}}{\left(K_{1}+\frac{1}{\tau_{1}}+\frac{1}{\tau_{2}}\right)^{2}}$.

The inequality $t_{1} \geq 0$ straightforwardly comes from the non-negativity of the model parameters. In particular, $t_{1}=0$ if and only if $K_{1}=0$ or $K_{2}=0$ or $\rho=0$ (as well 
as it approaches zero if and only if $K_{1}$ tends to infinity or one parameter among $\tau_{1}, \tau_{2}$ approaches zero). On the other hand, the second term of $x$ satisfies the inequality $-1 \leq t_{2} \leq 0$. The non-positivity of $t_{2}$ trivially comes again from the nonnegativity of the model parameters; conversely, the inequality $t_{2} \geq-1$ holds if and only if $\left(K_{1}+1 / \tau_{1}-1 / \tau_{2}\right)^{2} \geq 0$, which is trivially satisfied for any parameter setting. Moreover, the minimum value $t_{2}=-1$ is obtained if and only if the condition $K_{1}+1 / \tau_{1}-1 / \tau_{2}=0$ holds. So, from the previous arguments we can conclude that

$x=t_{1}+t_{2} \geq 0-1=-1$.

We also notice that

$x=-1, \Longleftrightarrow\left\{\begin{array}{l}K_{1}=0 \text { or } K_{2}=0 \text { or } \rho=0, \\ \text { and } \\ K_{1}+1 / \tau_{1}-1 / \tau_{2}=0 .\end{array}\right.$

Finally, from property (14) and Eqs. (10), we easily get the following items:

- $\lambda_{1}$ is always real and negative (for any $x \geq-1$ ),

- $\lambda_{2}$ is always real; moreover, it is $\lambda_{2}>0$ for $x>0$ and $\lambda_{2} \leq 0$ for $-1 \leq x \leq 0$,

that complete the proof of the theorem.

The results on the growing dynamics of system (1) given above are summarized by the flow scheme of Figure 3, showing the possible combinations of working modes $\left(\mu_{1}, \mu_{2}\right)$ and growing dynamics $(g, \bar{g})$, on the basis of $R(t) / P(t)$ and $x$ values. The figure shows that the population of ribosomes and proteins can actually grow only when $x>0$, but it depends on the value of the ratio $R(t) / P(t)$ : the growth dynamics $g$ is actually obtained only under the working mode $\mu_{2}$, i.e. when $R / P<\rho$ (green block). Conversely, when $-1 \leq x \leq 0$ the growth is not allowed, independently of the values of the state variables (i.e. of the current working mode).

Let us now provide some properties on the switch between the two working modes $\mu_{1}$ and $\mu_{2}$. In general, a piecewise affine system can show different behavior, spanning from stability to chaos [23]. The following results give conditions on the model parameters determining if each working mode remains stable or switches to the other one. Being in a given working mode at time $t$ only depends on the ratio $R(t) / P(t)$ at the same time, but the value of the model parameters is the only knowledge that we need in order to determine if the working mode is stable, i.e. it is indefinitely maintained after $t$, or if it is unstable, i.e. a switch towards the opposite working mode soon or later will occur.

Let us study such properties for meaningful values of the model parameters, that is for positive values. Indeed, when some parameters vanish either the model is not defined or the switching mechanism has no meaning because no growth can be accomplished (the condition $x>0$ is straightforwardly violated). In particular, the dynamical equations of (1) are not defined when $\tau_{1}=0$ or $\tau_{2}=0$. Conversely, according to definition (9), it is $x<0$ when $K_{1}=0$ or $K_{2}=0$ or $\rho=0$, meaning that ribosomes and proteins are not growing under such parameter conditions (see 


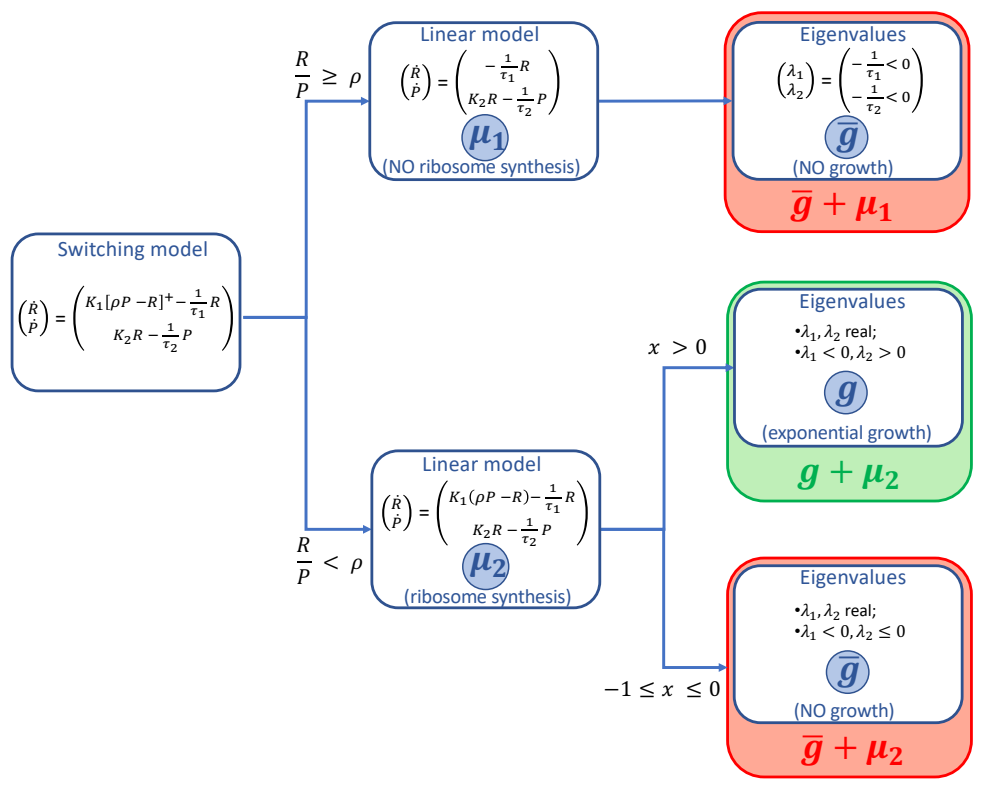

Figure 3

Possible combinations of working modes and growing dynamics.

Theorem 1). We finally notice that the condition $x>-1$ holds when only positive values of the model parameters are considered (indeed, according to relation (15), at least one of the parameters $K_{1}, K_{2}, \rho$ must vanish in order to obtain $x=-1$ ).

Let us first give the result establishing whether the ratio $R(t+\delta) / P(t+\delta)$ remains larger than/equal to $\rho$ (no synthesis, $\mu_{1}$ ), or alternatively becomes lower than $\rho$ (synthesis, $\mu_{2}$ ), for $\delta \rightarrow \infty$.

Theorem 2. Given the condition $R(t) / P(t) \geq \rho$ at a given time $t$, the ribosome synthesis of system (1) is not active and the working mode $\mu_{1}$ is going to change or not in $t+\delta$, for $\delta \rightarrow \infty$, only depending on the model parameters. In particular,

1. the working mode $\mu_{1}$ sooner or later will switch to $\mu_{2}$ if

$$
\tau_{1} \leq \tau_{2}
$$

or if

$\tau_{1}>\tau_{2} \quad$ and $\quad \rho K_{2}>1 / \tau_{2}-1 / \tau_{1}$

2. otherwise the working mode $\mu_{1}$ is indefinitely maintained.

Proof. The proof of Theorem 2 is completely given in Section IV.A of [3].

From Theorem 2 the following corollary can be derived. 
Corollary. If the model parameters are such that $\rho K_{2}>1 / \tau_{2}-1 / \tau_{1}$, then the switch $\mu_{1} \rightarrow \mu_{2}$ is unavoidable; otherwise it is forbidden.

Proof. The proof of the corollary straightforwardly comes noticing that the condition $\tau_{1} \leq \tau_{2}$ necessarily implies $\rho K_{2}>1 / \tau_{2}-1 / \tau_{1}$, as $1 / \tau_{2}-1 / \tau_{1} \leq 0$ and $\rho, K_{2}>0$. So, the condition $\rho K_{2}>1 / \tau_{2}-1 / \tau_{1}$ is the largest condition on the parameter values implying that the switch $\mu_{1} \rightarrow \mu_{2}$ sooner or later will happen, independently of the values of $\tau_{1}, \tau_{2}$.

The next theorem deals with the behaviour of $R(t+\delta) / P(t+\delta)$ for $\delta \rightarrow \infty$, starting from $R(t) / P(t)<\rho$ (presence of synthesis, $\mu_{2}$ ).

Theorem 3. Given the condition $R(t) / P(t)<\rho$ at a given time $t$, the ribosome synthesis of system (1) is active and the working mode $\mu_{2}$ is going to change or not in $t+\delta$, for $\delta \rightarrow \infty$, only depending on the model parameters. In particular,

1. the working mode $\mu_{2}$ is indefinitely maintained if

$x>0, \quad($ growth condition $)$

or if

$-1<x \leq 0 \quad$ and $\quad \rho K_{2} \geq 1 / \tau_{2}-1 / \tau_{1} ;$

2. otherwise the working mode $\mu_{2}$ sooner or later will switch to $\mu_{1}$.

Proof. The proof of the stability of $\mu_{2}$ when the growth condition $x>0$ holds is given in Section IV.B of [3]. Here we extend such results to include also the case $-1<x \leq 0$.

In order to study the behaviour of the ratio $R(t+\delta) / P(t+\delta), \delta \rightarrow \infty$, when $-1<$ $x \leq 0$ and $R(t) / P(t)<\rho$, we need to compute the explicit solutions of system (3). Such solutions in $t+\delta$ can be given as linear combinations of the natural modes $e^{\lambda_{i} \delta}, i=1,2$, where the eigenvalues $\lambda_{i}$ are provided by Eqs. (10). In particular, we have:

$\left[\begin{array}{l}R(t+\delta) \\ P(t+\delta)\end{array}\right]=u_{1} v_{1}^{T}\left[\begin{array}{l}R(t) \\ P(t)\end{array}\right] e^{\lambda_{1} \delta}+u_{2} v_{2}^{T}\left[\begin{array}{l}R(t) \\ P(t)\end{array}\right] e^{\lambda_{2} \delta}$,

where $u_{i}$ and $v_{i}$ are respectively the right and the left eigenvectors associated to the eigenvalues $\lambda_{i}, i=1,2$ (i.e. the solutions of the systems $\left(A-\lambda_{i} I\right) u_{i}=0, v_{i}^{T}(A-$ $\left.\left.\lambda_{i} I\right)=0, i=1,2\right)$. Recalling the expression of the eigenvectors reported in [3], we obtain:

$$
\begin{aligned}
R(t+\delta)= & \frac{\lambda_{1}+\frac{1}{\tau_{2}}}{\lambda_{2}-\lambda_{1}}\left(-R(t)+\frac{\lambda_{2}+\frac{1}{\tau_{2}}}{K_{2}} P(t)\right) e^{\lambda_{1} \delta} \\
& +\frac{\lambda_{2}+\frac{1}{\tau_{2}}}{\lambda_{2}-\lambda_{1}}\left(R(t)-\frac{\lambda_{1}+\frac{1}{\tau_{2}}}{K_{2}} P(t)\right) e^{\lambda_{2} \delta}
\end{aligned}
$$




$$
\begin{aligned}
P(t+\delta)= & \frac{K_{2}}{\lambda_{2}-\lambda_{1}}\left(-R(t)+\frac{\lambda_{2}+\frac{1}{\tau_{2}}}{K_{2}} P(t)\right) e^{\lambda_{1} \delta} \\
& +\frac{K_{2}}{\lambda_{2}-\lambda_{1}}\left(R(t)-\frac{\lambda_{1}+\frac{1}{\tau_{2}}}{K_{2}} P(t)\right) e^{\lambda_{2} \delta}
\end{aligned}
$$

According to the expression of eigenvalues (10) and to the condition $-1<x \leq 0$, by also recalling the relations implied by the minimal condition $x=-1$ given by (15), it is easy to obtain the following inequalities:

$$
\begin{aligned}
& \lambda_{1}<\lambda_{2} \leq 0 \quad \Longrightarrow \quad e^{\lambda_{1} \tau}<e^{\lambda_{2} \tau} \leq 1, \quad \forall \tau>0, \\
& \lambda_{2}-\lambda_{1}=2 y \sqrt{1+x}>0, \\
& \lambda_{1}+\frac{1}{\tau_{2}}<0, \quad \lambda_{2}+\frac{1}{\tau_{2}}>0 .
\end{aligned}
$$

Inequalities (23) can be exploited to verify that the solutions of $R(t+\delta)$ and $P(t+\delta)$ given by Eqs. (21)-(22) are strictly positive for any pair $R(t), P(t)>0$ and that their behaviours tend to be equal to the following ones for $\delta \rightarrow \infty$

$$
\begin{aligned}
& R(t+\delta)=\frac{\lambda_{2}+\frac{1}{\tau_{2}}}{\lambda_{2}-\lambda_{1}}\left(R(t)-\frac{\lambda_{1}+\frac{1}{\tau_{2}}}{K_{2}} P(t)\right) e^{\lambda_{2} \delta}>0, \\
& P(t+\delta)=\frac{K_{2}}{\lambda_{2}-\lambda_{1}}\left(R(t)-\frac{\lambda_{1}+\frac{1}{\tau_{2}}}{K_{2}} P(t)\right) e^{\lambda_{2} \delta}>0,
\end{aligned}
$$

since the natural mode $e^{\lambda_{1} \delta}$ tends to zero more rapidly than $e^{\lambda_{2} \delta}$ (see inequalities (23)). This implies that the limit of the ratio $R(t+\delta) / P(t+\delta)$ is given by the following expression

$\lim _{\delta \rightarrow \infty} \frac{R(t+\delta)}{P(t+\delta)}=\frac{\lambda_{2}+\frac{1}{\tau_{2}}}{K_{2}} \triangleq \gamma_{2}>0$.

Studying the sign of the time derivative of $R(t+\delta) / P(t+\delta)$, it is possible to prove its monotonic behaviour in approaching $\gamma_{2}$. Indeed, by introducing the quantity $\gamma_{1} \triangleq-\left(\lambda_{1}+1 / \tau_{2}\right) / K_{2}$, from Eqs. (21)-(22) we have

$$
\frac{R(t+\delta)}{P(t+\delta)}=\frac{\gamma_{1}\left(R(t)-\gamma_{2} P(t)\right) e^{\lambda_{1} \delta}+\gamma_{2}\left(R(t)+\gamma_{1} P(t)\right) e^{\lambda_{2} \delta}}{-\left(R(t)-\gamma_{2} P(t)\right) e^{\lambda_{1} \delta}+\left(R(t)+\gamma_{1} P(t)\right) e^{\lambda_{2} \delta}} .
$$

By exploiting the time derivative:

$$
\frac{d}{d \delta}\left[\frac{R(t+\delta)}{P(t+\delta)}\right]=\frac{\frac{P(t)^{2}}{K_{2}}\left(\gamma_{2}-\frac{R(t)}{P(t)}\right)\left(\frac{R(t)}{P(t)}+\gamma_{1}\right)\left(\lambda_{2}-\lambda_{1}\right)^{2}}{\left(-\left(R(t)-\gamma_{2} P(t)\right) e^{\lambda_{1} \delta}+\left(R(t)+\gamma_{1} P(t)\right) e^{\lambda_{2} \delta}\right)^{2}} e^{\left(\lambda_{1}+\lambda_{2}\right) \delta}
$$

Taking into account inequalities (23), it is easy to verify that the sign of (27) only depends on the value of $R(t) / P(t)$, i.e. on the sign of the factor $\gamma_{2}-R(t) / P(t)$. Such 
a sign cannot change with $\delta$ and the monotonic behaviour of $R(t+\delta) / P(t+\delta)$ is guaranteed for any value of the initial ratio $R(t) / P(t)$.

In order to complete the proof we need to find the parameter conditions under which the relation $\gamma_{2} \leq \rho$, as well as the opposite one, is satisfied. Indeed, since $R(t) / P(t)<\rho$, requiring $\gamma_{2} \leq \rho$ means that the ratio of ribosomes over proteins will remain lower than $\rho$ for any finite time, being $\gamma_{2}$ the limit value of the ratio for infinite time. So, by imposing the relation

$\gamma_{2}=\frac{\lambda_{2}+\frac{1}{\tau_{2}}}{K_{2}} \leq \rho$

we have

$\lambda_{2} \leq K_{2} \rho-\frac{1}{\tau_{2}}$,

where $\lambda_{2} \leq 0$ because of the condition $-1<x \leq 0$. Now, substituting the expression of $\lambda_{2}$ given in (10) in the previous inequality and performing simple algebraic operations, it is possible to obtain the final relation

$K_{2} \rho+\frac{1}{\tau_{1}}-\frac{1}{\tau_{2}} \geq 0$

meaning that

$\gamma_{2} \leq \rho \quad \Leftrightarrow \quad K_{2} \rho+\frac{1}{\tau_{1}}-\frac{1}{\tau_{2}} \geq 0$.

Obviously, implications (31) can be also given exploiting the opposite inequalities, i.e.

$\gamma_{2}>\rho \quad \Leftrightarrow \quad K_{2} \rho+\frac{1}{\tau_{1}}-\frac{1}{\tau_{2}}<0$.

So, we can conclude that, when $-1<x \leq 0$, the ratio $R(t+\delta) / P(t+\delta)$ remains under the threshold $\rho$ for $\delta \rightarrow \infty$ if $K_{2} \rho \geq 1 / \tau_{2}-1 / \tau_{1}$ or it crosses the threshold otherwise.

From Theorem 3 the following corollary can be derived.

Corollary. If the model parameters are such that $\rho K_{2}<1 / \tau_{2}-1 / \tau_{1}$, then the switch $\mu_{2} \rightarrow \mu_{1}$ is unavoidable; otherwise it is forbidden.

Proof. The proof comes from Theorem 3 noticing that the inequality $\rho K_{2} \geq 1 / \tau_{2}-$ $1 / \tau_{1}$ can be assumed as the only condition for the stability of $\mu_{2}$, independently of the value of $x$. Indeed, the growth condition $x>0$ can be rewritten as

$K_{1} K_{2} \rho>\left(K_{1}+\frac{1}{\tau_{1}}\right) \frac{1}{\tau_{2}}$ 
or equivalently, for positive values of $K_{1}$, as

$K_{2} \rho>\frac{K_{1}+\frac{1}{\tau_{1}}}{K_{1}} \frac{1}{\tau_{2}}$.

From condition (34) and from the positivity of the model parameters we obtain the following chain of inequalities

$K_{2} \rho>\frac{K_{1}+\frac{1}{\tau_{1}}}{K_{1}} \frac{1}{\tau_{2}}>\frac{1}{\tau_{2}}>\frac{1}{\tau_{2}}-\frac{1}{\tau_{1}}$,

showing that $x>0$ implies $\rho K_{2}>1 / \tau_{2}-1 / \tau_{1}$. So, the condition $\rho K_{2} \geq 1 / \tau_{2}-1 / \tau_{1}$ can be taken as the only constraint to be verified in order to establish the stability of $\mu_{2}$, independently of the value of $x$.

The results of the corollaries of Theorems 2 and 3 are depicted in Figure 4, where all the possible working modes and growing dynamics of system (1) are represented in the positive orthant of the parameter space, showing when the transitions are forbidden or unavoidable.

\section{Positive parameter space}

$(x>-1)$

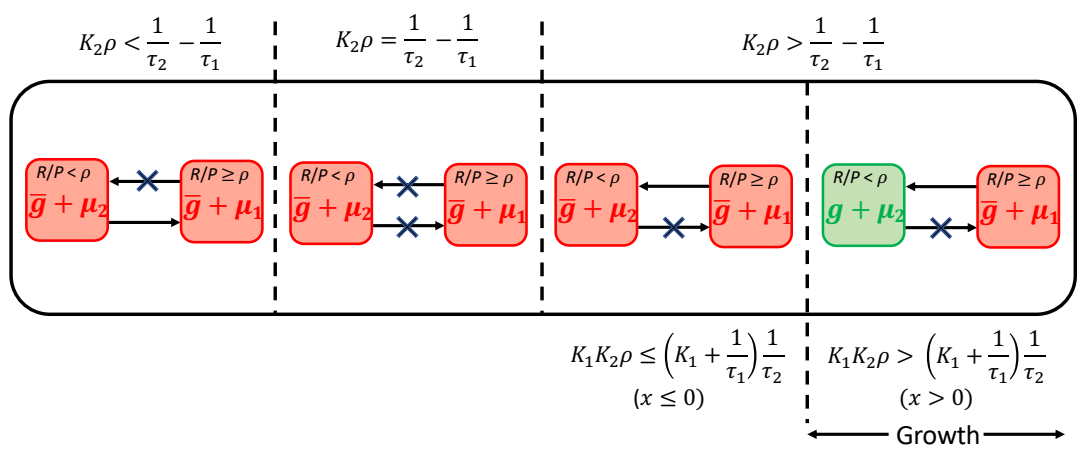

Figure 4

Switching of the working modes in the parameter space.

\section{Properties of the cell cycle module}

In 1977 Hartwell and Unger proposed in [15] a relationship that links the daughter and parent cycle period, $T_{D}$ and $T_{P}$, to the growth rate of a budding yeast cell, namely the positive eigenvalue $\lambda_{2}$ in our growth model (1):

$e^{-\lambda_{2} T_{D}}+e^{-\lambda_{2} T_{P}}=1$

where $T_{D}=T_{1}(\mathscr{D})+T_{2}+T_{B}$ and $T_{P}=T_{1}(\mathscr{P})+T_{2}+T_{B}$. 
The relationship can be derived from a minimal model of exponentially growing yeast populations that comprises only 2 cell types (parents and daughters of genealogical age 1) in either budded or unbudded state. The equation has been shown to be satisfied in exponentially growing cells $[16,24]$. Constraint (36) is graphically represented by the mesh in Figure 5, where the Mass Duplication Time $(M D T)$ is exploited instead of $\lambda_{2}$, on the basis of the relation $M D T=\ln (2) / \lambda_{2}$.

The remainder of this section is devoted to derive and extend analogous relationships among the cycle periods of the proposed growth and cycle cell model, that accounts for many kinds of daughter and parent cells, according to the rules that:

- a daughter cell of any genealogical age, $\mathscr{D}_{k}$, provides at cellular division a pair of newborn $\mathscr{D}_{1}$ and $\mathscr{P}_{1}$ cells;

- a parent cell of genealogical age $k, \mathscr{P}_{k}$, provides at cellular division a pair of newborn $\mathscr{D}_{k+1}$ and $\mathscr{P}_{k+1}$ cells.

Further rules involve the facts that:

- proteins and ribosomes produced during the budded phase go exclusively to the bud (the future daughter cell);

- $T_{2}$ and $T_{B}$ are (in average) the same for daughter and parents of any genealogical age;

- cells are supposed to be in balanced exponential growth, with growth rate provided by $\lambda_{2}$;

- parent cells grow at a slower growth rate during their unbudded phase, due to the mechanical stress discussed in Section 3. The growth rate associated to $\mathscr{P}_{k}$ cells in G1-phase will be denoted in the following as $\lambda_{2 k}$ and it can be computed from the model parameters exploiting the same function used for $\lambda_{2}$, i.e. Eq. (10), but assuming that the mechanical stress influences the values of the parameters related to the protein dynamics. In other words, $\lambda_{2 k}$ will change for $\mathscr{P}_{k}$ cells in G1-phase by changing the age $k$ because of the relation $\lambda_{2 k}=\lambda_{2 k}\left(K_{2 k}, \tau_{2 k}\right)$.

Denoting by $T_{G 1}$ the length of the $\mathrm{G} 1$ phase $\left(T_{G 1}=T_{1}+T_{2}\right)$, the first relationship is derived taking into account the exponential growth of a $\mathscr{D}_{1}$ cell from birth to bud:

$P_{0}\left(\mathscr{D}_{1}\right) e^{\lambda_{2} T_{G 1}\left(\mathscr{D}_{1}\right)}=P_{S}\left(\mathscr{D}_{1}\right)$,

and from bud to cellular division, assuming the newborn $\mathscr{D}_{1}$ cell to be identical to the original $\mathscr{D}_{1}$ :

$P_{s}\left(\mathscr{D}_{1}\right) e^{\lambda_{2} T_{B}}=P_{c d}\left(\mathscr{D}_{1}\right)=P_{0}\left(\mathscr{P}_{1}\right)+P_{0}\left(\mathscr{D}_{1}\right)=P_{s}\left(\mathscr{D}_{1}\right)+P_{0}\left(\mathscr{D}_{1}\right)$,

so that:

$P_{S}\left(\mathscr{D}_{1}\right) e^{\lambda_{2} T_{B}}=P_{S}\left(\mathscr{D}_{1}\right)+P_{S}\left(\mathscr{D}_{1}\right) e^{-\lambda_{2} T_{G 1}\left(\mathscr{D}_{1}\right)}$,

and finally:

$e^{-\lambda_{2} T\left(\mathscr{D}_{1}\right)}+e^{-\lambda_{2} T_{B}}=1$. 
Notice that eq.(40) very well resembles eq. (36), and it can be represented again by the mesh of Figure 5. By explicitly accounting for $T\left(\mathscr{D}_{1}\right)=T_{1}\left(\mathscr{D}_{1}\right)+T_{2}+T_{B}$, a mesh can be drawn constraining the three timers lengths $\left(T_{1}\left(\mathscr{D}_{1}\right), T_{2}, T_{B}\right)$, for any given value of $\lambda_{2}$ :

$T_{1}\left(\mathscr{D}_{1}\right)=\frac{1}{\lambda_{2}} \ln \left(\frac{1}{e^{\lambda_{2} T_{B}}-1}\right)-T_{2}$.

In Figure 6 we report the case for $\lambda_{2}=0.0073 \mathrm{~min}^{-1}$, corresponding to the experimental condition of $M D T=97 \mathrm{~min}$ in glucose $2 \%$. It has to be stressed that, according to (41), not all the pairs $\left(T_{2}, T_{B}\right)$ allow a feasible (i.e. positive) choice for timer $T_{1}\left(\mathscr{D}_{1}\right)$ length. In fact, for given values of $T_{2}$, according to (41) it must be:

$$
T_{1}\left(\mathscr{D}_{1}\right)>0 \quad \rightarrow \quad T_{B}<\frac{1}{\lambda_{2}} \ln \left(1+e^{-\lambda_{2} T_{2}}\right) .
$$

Figure 7 shows the upper bound for $T_{B}$ as coming from (42) according to different values of $T_{2}$. The same reasoning can be generalized, this time taking into account the exponential growth of a cell $\mathscr{D}_{k}$ from birth to bud, so that:

$P_{0}\left(\mathscr{D}_{k}\right) e^{\lambda_{2} T_{G 1}\left(\mathscr{D}_{k}\right)}=P_{s}\left(\mathscr{D}_{k}\right)$,

and the exponential growth of a $\mathscr{P}_{k-1}$ cell from bud to cellular division:

$P_{s}\left(\mathscr{P}_{k-1}\right) e^{\lambda_{2} T_{B}}=P_{c d}\left(\mathscr{P}_{k-1}\right)=P_{0}\left(\mathscr{P}_{k}\right)+P_{0}\left(\mathscr{D}_{k}\right)=P_{s}\left(\mathscr{P}_{k-1}\right)+P_{0}\left(\mathscr{D}_{k}\right)$,

so that:

$P_{S}\left(\mathscr{P}_{k-1}\right) e^{\lambda_{2} T_{B}}=P_{S}\left(\mathscr{P}_{k-1}\right)+P_{S}\left(\mathscr{D}_{k}\right) e^{-\lambda_{2} T_{G 1}\left(\mathscr{D}_{k}\right)}$,

or equivalently:

$P_{s}\left(\mathscr{P}_{k-1}\right)=P_{s}\left(\mathscr{P}_{k-1}\right) e^{-\lambda_{2} T_{B}}+P_{s}\left(\mathscr{D}_{k}\right) e^{-\lambda_{2} T\left(\mathscr{D}_{k}\right)}$.

On the other hand, if we consider the exponential growth of a $\mathscr{P}_{k}$ cell from birth to bud, we have:

$P_{0}\left(\mathscr{P}_{k}\right) e^{\lambda_{2 k} T_{G 1}\left(\mathscr{P}_{k}\right)}=P_{s}\left(\mathscr{P}_{k}\right)=P_{S}\left(\mathscr{P}_{k-1}\right) e^{\lambda_{2 k} T_{G 1}\left(\mathscr{P}_{k}\right)}$,

since $P_{0}\left(\mathscr{P}_{k}\right)=P_{s}\left(\mathscr{P}_{k-1}\right)$. Notice that eq. (47) accounts for different growth rates for parent cells of different genealogical ages, due to the mechanical stress before the bud occurs. Differently from eqs. (40) and (36), constraints (45)-(47) involve size and cycle parameters together.

\section{Conclusions}

The coherence of a mathematical model (i.e. whether the associated solutions are meaningful for the largest range of the feasible model parameters) is an important feature a good model is required to attain, especially when aiming at describing a 


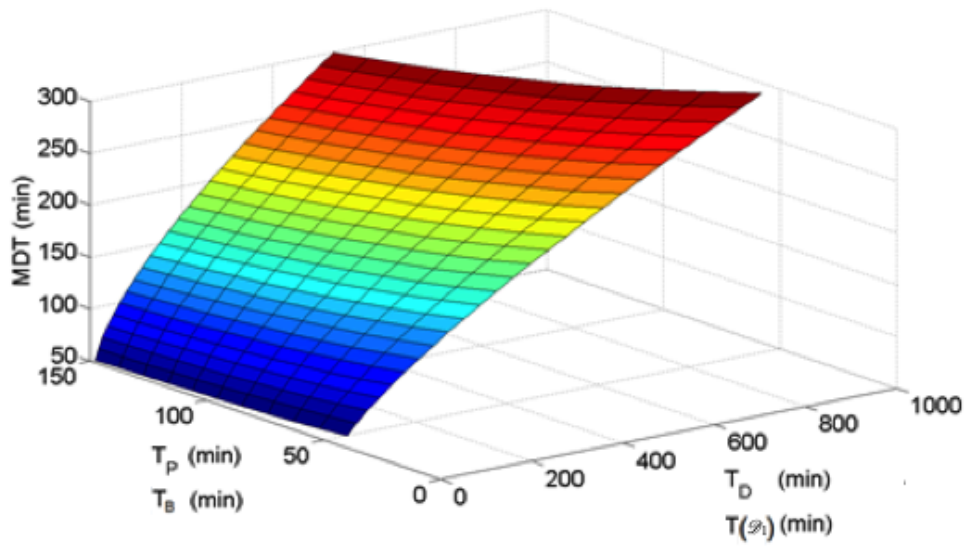

Figure 5

Constraint among $M D T$, parent and daughter cycle lengths ( $T_{P}$ and $T_{D}$, respectively). It is obtained from (36), exploiting also the relation $M D T=\ln (2) / \lambda_{2}$. The same mesh represents the relationship among $M D T$, budded phase and $\mathscr{D}_{1}$ cycle length $\left(T_{B}\right.$ and $T\left(\mathscr{D}_{1}\right)$, respectively) in our model, according to (40).

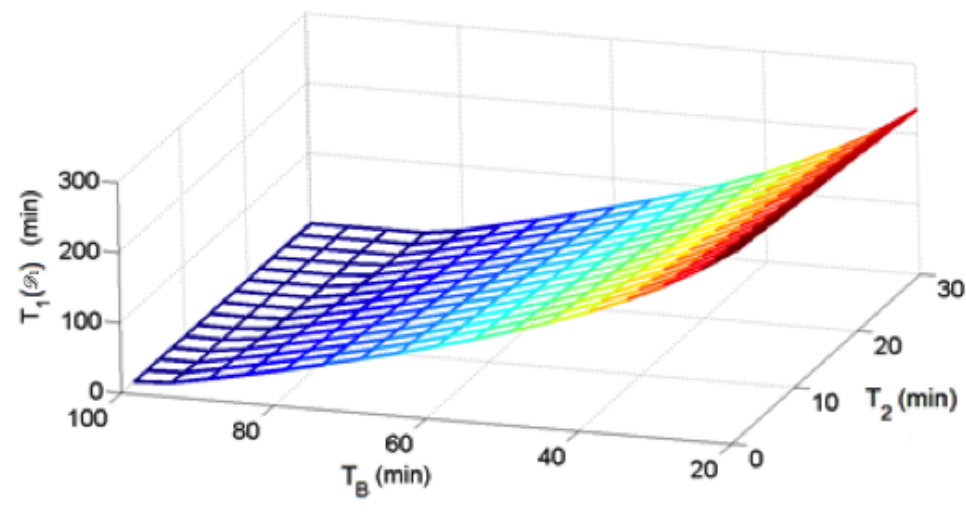

Figure 6

Constraint among $T_{1}\left(\mathscr{D}_{1}\right), T_{B}$ and $T_{2}$ for $M D T=97 \mathrm{~min}$, as coming from (41).

wide range of possible working modes. In this work we have investigated the qualitative behavior of a coarse-grain model of cellular growth, recently exploited as a module of a larger interconnected model that integrates metabolism, growth and cell cycle in yeast. More in details, we found a specific sufficient condition $(x>0)$ for the growth of ribosome and protein populations. In particular, when starting the dynamic evolution with active ribosome synthesis such a condition guarantees to maintain synthesis and growth for any time. Conversely, when starting with the ribosome synthesis initially inactive, determining a temporary non-growing state, the system approaches a state condition that allows the switch for an active synthesis and, consequently, exponential growth. On the other hands, if such a condition is 


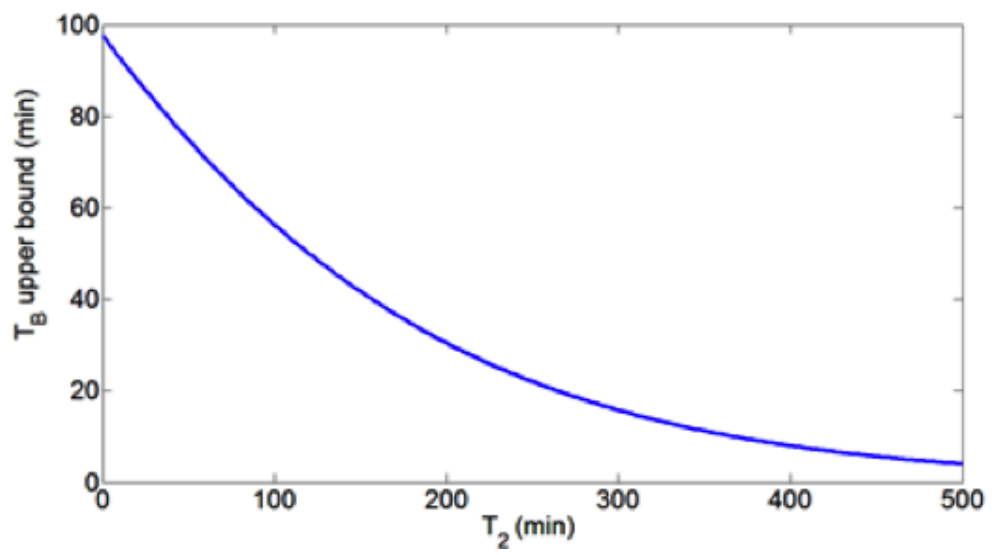

Figure 7

Upper bound for $T_{B}$ as coming from (42) according to different values of $T_{2}$.

violated $(x \leq 0)$, no growth is possible, independently of the ribosome synthesis. Furthermore, by linking the growth module to a basic set of timers describing the cell cycle, we are able to derive constraints among timers and growth rate that somehow generalize, to the more complex case accounting for the variety of genealogical ages, analogous constraints achieved half a century ago and still exploited as a preliminary validity check for mathematical models of cell cycle in yeast.

\section{Acronyms}

For the convenience of the reader, the following table collects all the acronyms used in the text.

\begin{tabular}{|c|l|}
\hline Acronym & Definition \\
\hline \hline Whi5 & $\begin{array}{l}\text { Transcriptional regulator in the budding yeast cell cycle, notably } \\
\text { in the G1 phase. }\end{array}$ \\
\hline Clb5/6 & B-type S-phase cyclins in yeast that assist in cell cycle regulation. \\
\hline Sic1 & $\begin{array}{l}\text { Stoichiometric inhibitor of Cdk1-Clb complexes (bindings of the } \\
\text { Cyclin-dependent kinase 1 - a key player of the cell cycle } \\
\text { regulation in yeast - and the B-type cyclins Clb). }\end{array}$ \\
\hline TOR & Target Of Rapamycin, protein kinase \\
\hline Sfp1 & Transcription factor that regulates growth and cell division in yeast. \\
\hline ppGpp & $\begin{array}{l}\text { Guanosine tetraphosphate, an alarmone which is involved in the } \\
\text { stringent response in bacteria, causing the inhibition of RNA } \\
\text { synthesis when there is a shortage of amino acids present. }\end{array}$ \\
\hline
\end{tabular}

List of the acronyms used in the text. 


\section{Acknowledgement}

The institutional financial support of SYSBIO.ISBE.IT within the Italian Roadmap for ESFRI Research Infrastructures is gratefully acknowledged.

\section{References}

[1] S. Di Talia, J. M. Skotheim, J. M. Bean, E. D. Siggia, and F. R. Cross. The effects of molecular noise and size control on variability in the budding yeast cell cycle. Nature, 448:947-951, 2007.

[2] P. Palumbo, M. Vanoni, F. Papa, S. Busti, M. Wortel, B. Teusink, and L. Alberghina. An integrated model quantitatively describing metabolism, growth and cell cycle in budding yeast. In M. Pelillo et al., editors, Artificial Life and Evolutionary Computation, volume 830, pages 165-180. Springer, 2018. Communications in Computer and Information Science (CCIS book series).

[3] P. Palumbo, F. Papa, M. Vanoni, and L. Alberghina. Qualitative behavior of a coarse-grain growth model. In 23rd IEEE International Conference on Intelligent Engineering Systems, 2019.

[4] L. Alberghina, L. Mariani, and E. Martegani. Cell cycle modelling. Biosystems, 19:23-44, 1986.

[5] J. J. Turner, J. C. Ewald, and J. M. Skotheim. Cell size control in yeast. Current Biology, 22:R350-359, 2012.

[6] E. Martegani, L. Popolo, L. Alberghina, and E. Sturani. Reduction of ribosome activity and synthesis of stable RNA in Neurospora crassa. Biochimica et Biophysica Acta, 610:318-330, 1980.

[7] M. Nomura, R. Gourse, and G. Baughman. Regulation of the synthesis of ribosomes and ribosomal components. Annual Review of Biochemistry, 53:75$117,1984$.

[8] E. Bosdriesz, D. Molenaar, B. Teusink, and F. J. Bruggeman. How fastgrowing bacteria robustly tune their ribosome concentration to approximate growth-rate maximization. The FEBS Journal, 282:2029-2044, 2015.

[9] V. Zinzalla, D. Stracka, W. Oppliger, and M. N. Hall. Activation of mTORC2 by association with the ribosome. Cell, 144:757-768, 2011.

[10] M. Cook and M. Tyers. Size control goes global. Current Opinion in Biotechnology, 18:341-350, 2007.

[11] K. W. Boehlke and J. D. Friesen. Cellular content of ribonucleic acid and protein in Saccharomyces cerevisiae as a function of exponential growth rate: calculation of the apparent peptide chain elongation rate. Journal of Bacteriology, 121:429-433, 1975.

[12] C. Waldron and F. Lacroute. Effect of growth rate on the amounts of ribosomal and transfer ribonucleic acids in yeast. Journal of Bacteriology, 122:855-865, 1975. 
[13] C. Waldron, R. Jund, and F. Lacroute. Evidence for a high proportion of inactive ribosomes in slow-growing yeast cells. Biochemical Journal, 168:409415, 1977.

[14] L. Alberghina and E. Sturani. Control of growth and of the nuclear division cycle in Neurospora crassa. Microbiological Reviews, 45:99-122, 1981.

[15] L. H. Hartwell and M. W. Unger. Unequal division in Saccharomyces cerevisiae and its implications for the control of cell division. The Journal of Cell Biology, 75:422-435, 1977.

[16] D. Porro, M. Vai, M. Vanoni, L. Alberghina, and C. Hatzis. Analysis and modeling of growing budding yeast populations at the single cell level. Cytometry A, 75:114-120, 2009.

[17] A. Lorincz and B. L. A. Carter. Control of cell size at bud initiation in Saccharomyces cerevisiae. Journal of general microbiology, 113:287-295, 1979.

[18] B. L. Carter and M. N. Jagadish. The relationship between cell size and cell division in the yeast Saccharomyces cerevisiae. Experimental Cell Research, 1978:112, 112.

[19] C. L. Woldringh, P. G. Huls, and N. O. Vischer. Volume growth of daughter and parent cells during the cell cycle of Saccharomyces cerevisiae a/alpha as determined by image cytometry. Journal of Bacteriology, 175:3174-3181, 1993.

[20] G. C. Johnston, C. W. Ehrhardt, A. Lorincz, and B. L. Carter. Regulation of cell size in the yeast Saccharomyces cerevisiae. Journal of Bacteriology, 137:1-5, 1979.

[21] L. Alberghina, Vai M., and M. Vanoni. Probing control mechanisms of cell cycle and ageing in budding yeast. Current Genomics, 5(8):615-627, 2004.

[22] D. E. Levin. Regulation of cell wall biogenesis in Saccharomyces cerevisiae: the cell wall integrity signaling pathway. Genetics, 189:1145-1175, 2011.

[23] R. Csikja, B. M. Garay, and J. Tóth. Chaos via two-valued interval maps in a piecewise affine model example for hysteresis. In Proceedings of the 19th International Symposium on Mathematical Theory of Networks and Systems-MTNS, pages 187-194, 2010.

[24] M. Vanoni, M. Vai, L. Popolo, and L. Alberghina. Structural heterogeneity in populations of the budding yeast Saccharomyces cerevisiae. Journal of Bacteriology, 156:1282-1291, 1983. 